\title{
DECOMPOSING TEXTURES USING EXPONENTIAL ANALYSIS
}

\author{
Yuan Hou ${ }^{1}$, Annie Cuyt ${ }^{1,3}$, Wen-shin Lee ${ }^{2}$ and Deepayan Bhowmik ${ }^{2}$ \\ ${ }^{1}$ Department of Computer Science, University of Antwerp, 2020 Antwerp, Belgium \\ ${ }^{2}$ Division of Computing Science \& Mathematics, University of Stirling, Stirling, FK9 4LA, UK \\ ${ }^{3}$ College of Mathematics and Statistics, Shenzhen University, Shenzhen, Guangdong 518060, China \\ \{yuan.hou, annie.cuyt\}@uantwerpen.be \\ \{wen-shin.lee, deepayan.bhowmik\}astir.ac.uk
}

\begin{abstract}
Decomposition is integral to most image processing algorithms and often required in texture analysis. We present a new approach using a recent 2-dimensional exponential analysis technique. Exponential analysis offers the advantage of sparsity in the model and continuity in the parameters. This results in a much more compact representation of textures when compared to traditional Fourier or wavelet transform techniques. Our experiments include synthetic as well as real texture images from standard benchmark datasets. The results outperform FFT in representing texture patterns with significantly fewer terms while retaining RMSE values after reconstruction. The underlying periodic complex exponential model works best for texture patterns that are homogeneous. We demonstrate the usefulness of the method in two common vision processing application examples, namely texture classification and defect detection.
\end{abstract}

Index Terms - Exponential analysis, multivariate, image decomposition, texture analysis.

\section{INTRODUCTION}

Frequency decomposition is a fundamental but challenging inverse problem to most image and signal processing applications. Major approaches can be categorised into 1) template based convolution methods, e.g., Fourier [1], cosine [2] or wavelet transforms [3] and 2) data driven adaptive approaches, e.g., empirical mode decomposition (EMD) [4] or empirical wavelet transform (EWT) [5]. Template based transforms are considered to be rigid and rely on predetermined basis functions or frames that are agnostic of the input image. On the contrary, adaptive techniques are flexible and often provide a better representation of the data. One common challenge across all these methods is the model cardinality of the frequency domain representation, which is often dictated by the available data granularity. Also, the mentioned techniques do not exploit in any way the structure present in the available data, such as in texture data. This paper proposes a new image decomposition technique using the recent multivariate exponential analysis in [6], with the aim to decompose texture images using a minimal representation.

In the past few years, multidimensional exponential analysis has attracted considerable attention in computational mathematics as well as in signal processing. In the 1dimensional case, the Prony-like exponential analysis methods, such as matrix pencil [7], ESPRIT [8], TLS-Prony [9] have all been successfully applied in solving many practical problems. At the same time, several multi-dimensional versions of these Prony-like methods have been developed, e.g., [10-15]. However, due to complexity issues, until recently these methods were not very suitable to serve as a general tool for higher-dimensional decomposition.

In [6], a $d$-dimensional exponential model of $n$ terms can be recovered from $O((d+1) n)$ regularly collected samples, which is substantially less than other multi-dimensional Prony-like methods, where the sample usage and computational complexity explode exponentially. This opens a wealth of possibilities, including certain image processing applications. Texture is a fundamental component of any image and is encountered in most image analysis problems. Therefore, it is no surprise that it is a very intensively researched area [16].

This paper explores the use of the multivariate exponential analysis presented in [6] as a new image decomposition technique that can express regular texture patterns with substantially fewer parameters. The key focus of this work constitutes the mathematical formulation of a new decomposition technique validated on both synthetic and real images available from benchmark data sets. We also show usage scenarios by applying our technique in texture classification and defect detection. The main contributions of our work are:

- Formulation of multivariate exponential analysis as a new image decomposition tool,

- Sparse image representation and reconstruction with a limited number of terms, and

- Use of exponential analysis in texture classification and defect detection.

(C) 2021 IEEE. Personal use of this material is permitted. Permission from IEEE must be obtained for all other uses, in any current or future media, including reprinting/republishing this material for advertising or promotional purposes, creating new collective works, for resale or redistribution to servers or lists, or reuse of any copyrighted component of this work in other works. 


\section{EXPONENTIAL IMAGE ANALYSIS}

We approach image decomposition as a two-dimensional exponential analysis problem. That is, we seek to determine $n$ and retrieve $\alpha_{j}, \phi_{j x}, \phi_{j y} \in \mathbb{C}$ from as few evaluations of

$$
f(x, y)=\sum_{j=1}^{n} \alpha_{j} \exp \left(\phi_{j x} x+\phi_{j y} y\right),
$$

as possible, where $(x, y)$ is the location of a pixel and $f(x, y)$ the value at the corresponding pixel. When $f(x, y)$ is periodic such as in some textures, and can be decomposed as a linear combination of sine and cosine functions, then $\phi_{j x}$ and $\phi_{j y}$ are purely imaginary.

The method we present is based on the new sparse algorithms [6, 17] requiring only $O(3 n)$ samples to analyze (1). We now summarize the 2-d idea explained in [6]. How to combine this with the 1-d technique of [17] is further detailed in [18].

Let $\Delta=\left(\Delta_{x}, \Delta_{y}\right) \neq(0,0)$ and $\delta=\left(\delta_{x}, \delta_{y}\right) \neq(0,0)$ be linearly independent, with

$$
\begin{gathered}
\left|\Im\left(\phi_{j x} \Delta_{x}+\phi_{j y} \Delta_{y}\right)\right|<\pi, \quad j=1, \ldots, n, \\
\left|\Im\left(\phi_{j x} \delta_{x}+\phi_{j y} \delta_{y}\right)\right|<\pi, \quad j=1, \ldots, n,
\end{gathered}
$$

where $\Im(\cdot)$ denotes the imaginary part, and let the values $\exp \left(\phi_{j x} \Delta_{x}+\phi_{j y} \Delta_{y}\right), j=1, \ldots, n$ be mutually distinct. How to deal with non-distinct values is discussed in [6]. We sample $f(x, y)$ at the equidistant points $s \Delta$ and some shifted locations $s \Delta+\delta$ :

$$
\begin{aligned}
f_{s} & :=f\left(s \Delta_{x}, s \Delta_{y}\right), \quad s=0, \ldots, 2 n-1, \\
F_{s} & :=f\left(s \Delta_{x}+\delta_{x}, s \Delta_{y}+\delta_{y}\right), \quad s=0, \ldots, n-1 .
\end{aligned}
$$

Then first, the expressions $\exp \left(\phi_{j x} \Delta_{x}+\phi_{j y} \Delta_{y}\right), j=$ $1, \ldots, n$ are retrieved as the generalized eigenvalues $\lambda_{j}$ of

$$
\begin{aligned}
&\left(\begin{array}{cccc}
f_{1} & f_{2} & \cdots & f_{n} \\
f_{2} & \cdots & & f_{n+1} \\
\vdots & & & \vdots \\
f_{n} & f_{n+1} & \cdots & f_{2 n-1}
\end{array}\right) v_{j}= \\
& \\
& \lambda_{j}\left(\begin{array}{cccc}
f_{0} & f_{1} & \cdots & f_{n-1} \\
f_{1} & \cdots & & f_{n} \\
\vdots & & & \vdots \\
f_{n-1} & f_{n} & \cdots & f_{2 n-2}
\end{array}\right) v_{j}
\end{aligned}
$$

where the $v_{j}$ denote the right eigenvectors. Several numerical methods exist for the solution of this problem, among which [7. 8. 17) used in Section 3 Because of (2) we can uniquely retrieve the inner products

$$
\Phi_{j}:=\left\langle\phi_{j}, \Delta\right\rangle, \quad \phi_{j}=\left(\phi_{j x}, \phi_{j y}\right), \quad j=1, \ldots, n
$$

from the computed $\lambda_{j}=\exp \left(\Phi_{j}\right)$. We're not yet able to recover the individual $\phi_{j x}$ and $\phi_{j y}$ though.
Second, we rewrite the values $F_{s}$ as

$$
\begin{aligned}
F_{s} & =\sum_{j=1}^{n} \alpha_{j} \exp \left(\phi_{j x} \delta_{x}+\phi_{j y} \delta_{y}\right) \exp \left(s \Phi_{j}\right) \\
& =\sum_{j=1}^{n} A_{j} \exp ^{s}\left(\Phi_{j}\right), \quad A_{j}:=\alpha_{j} \exp \left(\phi_{j x} \delta_{x}+\phi_{j y} \delta_{y}\right)
\end{aligned}
$$

and we introduce the notations $\alpha:=\left(\alpha_{1}, \ldots, \alpha_{n}\right)^{T}, A:=$ $\left(A_{1}, \ldots, A_{n}\right)$. We solve the linear systems of interpolation conditions

$$
\left(\begin{array}{ccc}
1 & \cdots & 1 \\
\exp \left(\Phi_{1}\right) & \cdots & \exp \left(\Phi_{n}\right) \\
\vdots & & \vdots \\
\exp ^{2 n-1}\left(\Phi_{1}\right) & \cdots & \exp ^{2 n-1}\left(\Phi_{n}\right)
\end{array}\right) \alpha=\left(\begin{array}{c}
f_{0} \\
\vdots \\
f_{2 n-1}
\end{array}\right)
$$

and

$$
\left(\begin{array}{ccc}
1 & \cdots & 1 \\
\exp \left(\Phi_{1}\right) & \cdots & \exp \left(\Phi_{n}\right) \\
\vdots & & \vdots \\
\exp ^{n-1}\left(\Phi_{1}\right) & \cdots & \exp ^{n-1}\left(\Phi_{n}\right)
\end{array}\right) A=\left(\begin{array}{c}
F_{0} \\
\vdots \\
F_{n-1}
\end{array}\right)
$$

and define $\exp \left(\Psi_{j}\right):=A_{j} / \alpha_{j}=\exp \left(\left\langle\phi_{j}, \delta\right\rangle\right), j=1, \ldots, n$. Note that we have no problem to pair the $\Psi_{j}$ to the $\Phi_{j}, j=$ $1, \ldots, n$ since the $A_{j}$ are paired to the $\alpha_{j}, j=1, \ldots, n$ through the Vandermonde systems (4) and (5).

The fact that the vectors $\Delta$ and $\delta$ are linearly independent leads for each $j=1, \ldots, n$ to the regular linear system

$$
\left(\begin{array}{cc}
\Delta_{x} & \Delta_{y} \\
\delta_{x} & \delta_{y}
\end{array}\right)\left(\begin{array}{l}
\phi_{j x} \\
\phi_{j y}
\end{array}\right)=\left(\begin{array}{l}
\Phi_{j} \\
\Psi_{j}
\end{array}\right)
$$

from which the individual $\phi_{j x}$ and $\phi_{j y}$ can be obtained.

So all unknown parameters in (1) can be retrieved at the expense of $2 n$ evaluations $f_{s}$ and $n$ evaluations $F_{s}$, or a mere total of $3 n$ samples. In practice, when dealing with noisy reallife data, the value of $n$ is overestimated by $\eta>n$. Moreover, the minimal number of $3 \eta=2 \eta+\eta$ required samples for an $\eta$-term model of the form (1) is often again overestimated by $N+\mathfrak{n}$ with $N \geq 2 \eta$ and $\mathfrak{n} \geq \eta$. The square $n \times n$ generalized eigenvalue problem (3), the $2 n \times n$ Vandermonde system (4) and the $n \times n$ Vandermonde system (5) then respectively take the sizes $(N-\eta) \times \eta, N \times \eta$ and $\mathfrak{n} \times \eta$ and are all solved in the least squares sense.

As mentioned, we use a combination of the matrix pencil method studied in [7] with the rank reduction step described in [8]. We call this method the TLS-Prony method (as in [9]), since the first numerical method to perform exponential analysis was published by the French nobleman de Prony in $1795[19]$. 
We deal with $512 \times 512$ texture images and choose the following values for the parameters: $\Delta=(1,0), \delta \in$ $\{(0,1),(0,2), \ldots,(0,511)\}, n=33, \eta=170, N=512, \mathfrak{n}=$ 512 . This choice is merely inspired by the size of the image and the consideration that $\eta \approx N / 3$ works well for $(3)$. Also, the fact that 511 vertical shifts of the first image row are available, is exploited by letting the vector $\delta$ be any of the 511 vectors listed. Note that $\Psi_{j k}$ computed from $\delta_{k}=(0, k)$ relates to $\Psi_{j}$ computed from $\delta=(0,1)$ by $\exp \left(\Psi_{j k}\right)=\exp \left(\left\langle\phi_{j}, \delta_{k}\right\rangle\right)=\exp ^{k}\left(\Psi_{j}\right), k=1, \ldots, 511$.

The 512 different values $\exp ^{k}\left(\Psi_{j}\right), k=0, \ldots, 511$ obtained in this way constitute a Prony problem in their own right. We analyze them using the decimated Prony-type algorithm presented in [17], with a decimation factor equal to 4. The use of decimation allows to build in some validation of the result, which is useful as the $\exp ^{k}\left(\Psi_{j}\right), j=1, \ldots, n$ are in general less accurate than the $\exp \left(\Phi_{j}\right), j=1, \ldots, n$. The reason for this is that the various $\exp ^{k}\left(\Psi_{j}\right)$ are obtained from solutions of the rather sensitive Vandermonde systems (4) and (5). The chosen decimation factor also reduces the system size of $(3)$ from $(N-\eta) \times \eta$ to $75 \times 53$.

\section{EXPERIMENTAL RESULTS AND DISCUSSIONS}

To validate the proposed exponential analysis we perform experiments on both synthetic and real texture images and compare against fast Fourier transformation (FFT) results, thus demonstrating the use of sparsity in the frequency domain. We also apply our method in common vision applications that require texture analysis, e.g., texture classification and defect detection. Within the scope of this work, our model considers only homogeneous patterns across the image and therefore does not work effectively on textures with multiple patterns.

\subsection{Synthetic images}

Following model (1), we generate five synthetic textures each containing 11 terms. We compare the exponential analysis results against FFT (in each image FFT coefficients in magnitude below $10^{-2.5}$ of the largest FFT coefficient are discarded to reduce the model complexity). For both, these results are used to reconstruct the images and compute RMSE values. A selection is shown in Fig. 1 (columns 1-2) and Table 1 . The proposed exponential analysis can decompose images with a significantly reduced model complexity (more than $90 \%$ coefficient reduction against FFT) and still achieve a near perfect reconstruction (low RMSE). Details of all images, including parameters, and experimental results are available from cma.uantwerpen.be/publications

\subsection{Real texture images from benchmark dataset}

Encouraged by the nice results for the synthetic images, we also conduct experiments with samples from the standard
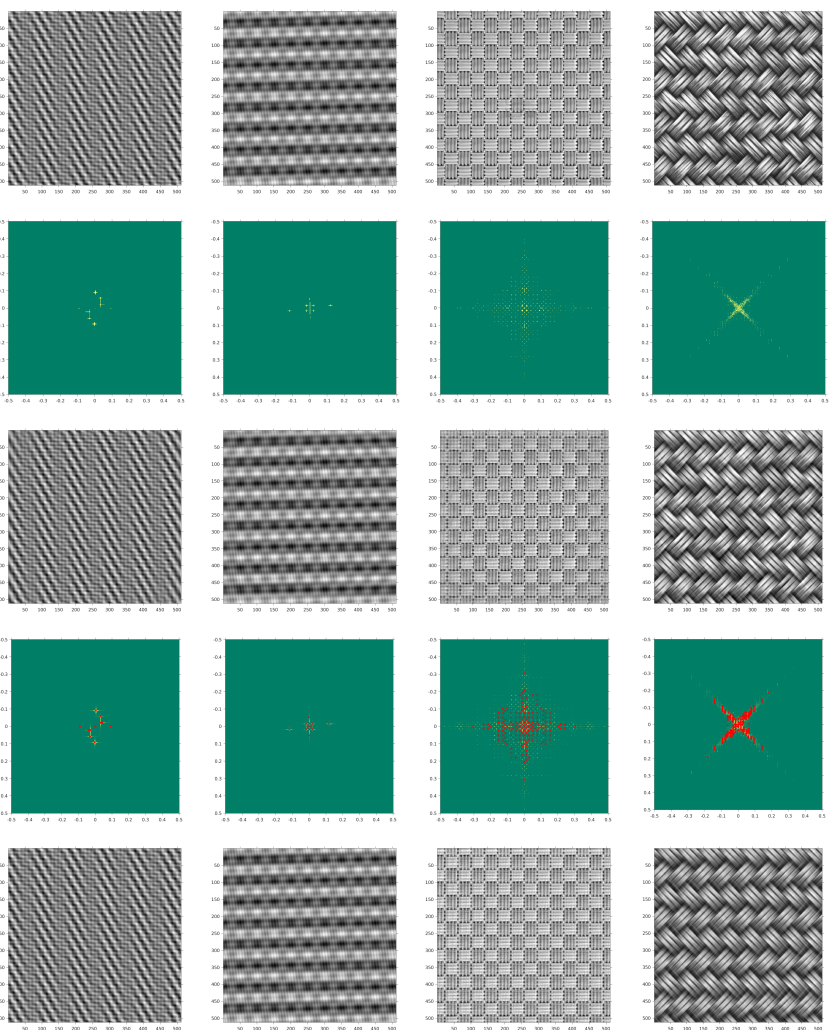

Fig. 1: Exponential analysis and comparison with FFT for synthetic images (columns 1-2) and real images (columns 34). Rows 1-5: original, thresholded FFT, reconstruction from thresholded FFT, exponential terms (• overlaid on FFT) and reconstruction from (1). Cardinality of representations given below rows (2) and (4).

Oxford Describable Textures Dataset (DTD, category: woven) [20]. Similar to Section 3.1], we compare the proposed methodology against the thresholded FFT in terms of number of coefficients and RMSE of the reconstructed image. The results are shown in Fig. 11 (columns 3-4) and Table 1 The proposed method outperforms the FFT representation with fewer terms while retaining the RMSE values. However, our method does not work well in woven_0064 or other images where the texture information is not sparse or consists of multiple patterns. This is due to the basic assumption of our model on sparsity and homogeneity, meaning periodicity as in (1).

\subsection{Use cases}

Finally, we present two vision applications that demonstrate the capability of our method: 1) texture classification and 2 ) texture defect detection. It is worth noting that within the scope of this work, the use cases do not consider a wider range of images and are limited to regular texture patterns. 


\begin{tabular}{c|c|c|c|c|c}
\hline & \multicolumn{2}{|c|}{ FFT } & \multicolumn{2}{c|}{ Ours } & Gain \\
\cline { 2 - 5 } & \# Terms & RMSE & \# Terms & RMSE & $(\%)$ \\
\hline \multicolumn{5}{c}{ Synthetic images } \\
\hline IM\#1 & 111 & 0.0169 & 11 & 0.0012 & $90 \%$ \\
IM\#2 & 231 & 0.0258 & 11 & 0.0015 & $95 \%$ \\
IM\#3 & 279 & 0.0285 & 11 & 0.0011 & $96 \%$ \\
IM\#4 & 163 & 0.0215 & 11 & 0.0011 & $93 \%$ \\
IM\#5 & 279 & 0.0235 & 11 & 0.0011 & $96 \%$ \\
\hline Real images from Oxford DTD dataset, category: woven \\
\hline 001 & 743 & 0.0254 & 246 & 0.0655 & $67 \%$ \\
003 & 793 & 0.0756 & 157 & 0.0924 & $80 \%$ \\
028 & 851 & 0.0513 & 141 & 0.0982 & $83 \%$ \\
038 & 1047 & 0.0685 & 239 & 0.1102 & $77 \%$ \\
064 & 4557 & 0.1100 & 256 & 0.2412 & $94 \%$ \\
\hline
\end{tabular}

Table 1: Comparison of image decomposition using FFT and proposed exponential analysis on synthetic and real images with respect to the number of terms to represent the texture, RMSE when reconstructed and \% gain in model reduction.

\subsubsection{Texture classification}

Texture analysis is fundamental to most vision applications. Texture representation and classification are essential parts of the processing pipeline and are researched widely [16]. Our argument is that using the proposed decomposition, texture can be represented compactly. In support, we experiment with a texture classification task and compare with typical speeded up robust features (SURF) [21]. In both cases the features are extracted to a vector which is passed to train a SVM classifier. Three example classes are prepared using samples from the DTD dataset, category: woven [20]. One unseen image from each category is used to predict the class. The results are shown in Table 2. For both methods (SURF and ours), the first two classes are correctly identified while Class-III is incorrectly predicted as Class-I. However, the number of features in our method is approximately 10 times less compared to SURF. This indicates the effectiveness of our work for many potential applications.

\subsubsection{Texture defect detection}

Defect detection in natural images is an important but challenging task in computer vision applications [22], including industrial material inspection and fabric production [23]. State-of-the-art algorithms often rely on machine learning, e.g., deep learning based approaches that require large training data sets. As an alternative approach, we propose exponential analysis to represent the texture with limited model complexity, reconstruct and compare with the input image. The image difference followed by a couple of morphological operations, such as erosion and dilation, identifies defects in

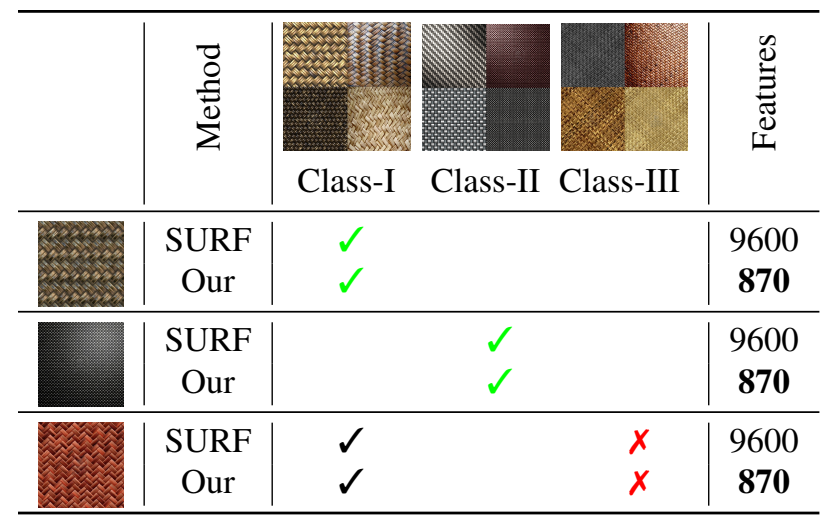

Table 2: Texture classification using exponential analysis and comparison with SURF. Classes I \& II are correctly identified. Col. 3-5: samples from the classes. Rows 2-4: test images.
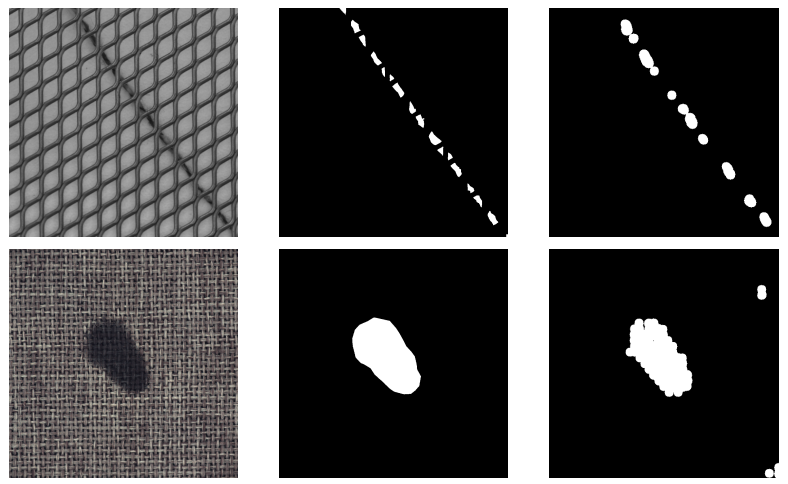

Fig. 2: Defect detection using exponential analysis: columns (1) original image, (2) ground truth and (3) detection.

test images (avaiable from [22], category: grid and carpet), as shown in Fig. 2. Our approach does not require any training data and hence will be of use to many applications of similar sort.

\section{CONCLUSION}

This paper proposes a new image decomposition method using 2-d exponential analysis. We exploit key properties of exponential analysis, such as sparsity in the data fitting problem and continuity in the frequency space. Such analysis results in a compact image representation in the frequency domain with significantly fewer coefficients. Experimental results outperform FFT for both synthetic and real texture images and indicate new opportunities in the image processing domain.

The usefulness of our method is illustrated with two common vision applications, texture classification and defect detection, which are central components of many image processing algorithms. Representing images with fewer terms will be beneficial for feature engineering in vision and can lead to better accuracy and a more efficient computation. 


\section{REFERENCES}

[1] Jae S. Lim, Two-Dimensional Signal and Image Processing, Prentice-Hall, Inc., USA, 1990.

[2] Gilbert Strang, "The discrete cosine transform," SIAM review, vol. 41, no. 1, pp. 135-147, 1999.

[3] Stéphane Mallat, A wavelet tour of signal processing, Elsevier, 1999.

[4] Jean Claude Nunes, Yasmina Bouaoune, Eric Delechelle, Oumar Niang, and $\mathrm{Ph}$ Bunel, "Image analysis by bidimensional empirical mode decomposition," Image and vision computing, vol. 21, no. 12, pp. 1019-1026, 2003.

[5] Jerome Gilles, "Empirical wavelet transform," IEEE transactions on signal processing, vol. 61, no. 16, pp. 3999-4010, 2013.

[6] Annie Cuyt and Wen-shin Lee, "Multivariate exponential analysis from the minimal number of samples," $A d v$. Comput. Math., vol. 44, pp. 987-1002, 2018, (Published online November 16, 2017).

[7] Yingbo Hua and Tapan K. Sarkar, "Matrix pencil method for estimating parameters of exponentially damped/undamped sinusoids in noise," IEEE Trans. Acoust., Speech, Signal Process., vol. 38, pp. 814-824, 1990.

[8] R. Roy and T. Kailath, "ESPRIT-estimation of signal parameters via rotational invariance techniques," IEEE Trans. Acoust., Speech, Signal Process., vol. 37, no. 7, pp. 984-995, July 1989.

[9] William M. Steedly, Ching-Hui J. Ying, and Randolph L. Moses, "Statistical analysis of TLS-based Prony techniques," Automatica J. IFAC, vol. 30, no. 1, pp. 115-129, 1994.

[10] Yingbo Hua, "Estimating two-dimensional frequencies by matrix enhancement and matrix pencil," IEEE Transactions on Signal Processing, vol. 40, no. 9, pp. 22672280, 1992.

[11] Stéphanie Rouguette and Mohamed Najim, "Estimation of frequencies and damping factors by two-dimensional ESPRIT type methods," IEEE Transactions on Signal Processing, vol. 49, no. 1, pp. 237-245, 2001.

[12] Swagata Nandi, Debasis Kundu, and Rajesh Kumar Srivastava, "Noise space decomposition method for twodimensional sinusoidal model," Comput. Statist. Data Anal., vol. 58, pp. 147-161, 2013.
[13] Thomas Peter, Gerlind Plonka, and Robert Schaback, "Prony's method for multivariate signals," Proc. Appl. Math. Mech., vol. 15, pp. 665-666, 2015.

[14] Stefan Kunis, Thomas Peter, Tim Römer, and Ulrich von der Ohe, "A multivariate generalization of Prony's method," Linear Algebra Appl., vol. 490, pp. 31-47, 2016.

[15] Tomas Sauer, "Prony's method in several variables," Numerische Mathematik, vol. 136, pp. 411-438, 2017.

[16] Li Liu, Jie Chen, Paul Fieguth, Guoying Zhao, Rama Chellappa, and Matti Pietikäinen, "From BoW to CNN: Two decades of texture representation for texture classification," International Journal of Computer Vision, vol. 127, no. 1, pp. 74-109, 2019.

[17] Matteo Briani, Annie Cuyt, Ferre Knaepkens, and Wenshin Lee, "VEXPA: Validated EXPonential Analysis through regular subsampling," Signal Processing, 2020, (Published online July 17, 2020).

[18] Annie Cuyt, Yuan Hou, Ferre Knaepkens, and Wenshin Lee, "Sparse multidimensional exponential analysis with an application to radar imaging," SIAM J. Scient. Comp., vol. 42, pp. B675-B695, 2020, (Published online May 14, 2020).

[19] R. de Prony, "Essai expérimental et analytique sur les lois de la dilatabilité des fluides élastiques et sur celles de la force expansive de la vapeur de l'eau et de la vapeur de l'alkool, à différentes températures," $J$. Ec. Poly., vol. 1, no. 22, pp. 24-76, 1795.

[20] Mircea Cimpoi, Subhransu Maji, Iasonas Kokkinos, Sammy Mohamed, and Andrea Vedaldi, "Describing textures in the wild," in Proc. IEEE CVPR, 2014, pp. 3606-3613.

[21] Herbert Bay, Tinne Tuytelaars, and Luc Van Gool, "SURF: Speeded up robust features," in Proc. European Conference on Computer Vision (ECCV). Springer, 2006, pp. 404-417.

[22] Paul Bergmann, Michael Fauser, David Sattlegger, and Carsten Steger, "MVTec AD-A comprehensive realworld dataset for unsupervised anomaly detection," in Proc. IEEE CVPR, 2019, pp. 9592-9600.

[23] Kazım Hanbay, Muhammed Fatih Talu, and Ömer Faruk Özgüven, "Fabric defect detection systems and methods-a systematic literature review," Optik, vol. 127, no. 24, pp. 11960-11973, 2016. 